\title{
Experiments on layer formation in stratified shear flow*
}

\author{
J. CISNEROS-AGUIRRE, J.L. PELEGRÍ, and P. SANGRÀ \\ Facultad de Ciencias del Mar, Universidad de Las Palmas de Gran Canaria, 35017 Las Palmas de Gran Canaria, \\ Canary Islands, Spain.
}

\begin{abstract}
SUMMARY: We discuss the experimental methodology and present the results of several experiments on the onset and growth of instabilities in stratified shear flow. Our results include the assessment of the effective diffusivity prior to the development of the Kelvin-Helmholtz $(\mathrm{K}-\mathrm{H})$ type instability and the measurement of the velocity field. This allows us to determine that the onset of the instability takes place when the Richardson number is close to 0.25 , this value slightly decreasing with increasing initial interface thickness. The formation of a fine layered structure at the interface is suggested by density profiles taken immediately after the generation of K-H billows, but the size of the full interface as well as the layers themselves decrease in the following few seconds. We hypothesize that the time scale of the K-H billows in our laboratory experiments is so short that there is no time for added mixing to further distribute and smoothen the initial layer structure.
\end{abstract}

Key words: stratified shear flow, Kelvin-Helmholtz instability, layer structure.

\section{INTRODUCTION}

Thorpe (1971) did the first detailed comparison of the theory of instability theory for stratified shear flow with experiments. His experiments consisted in characterizing the onset and growth of instabilities in tanks filled with two or more layers of fluid and subsequently tilted a small angle from the horizontal. In his seminal work Thorpe carefully studied the development and growth of Kelvin-Helmholtz (K$\mathrm{H})$ type disturbances, or class $\mathrm{C}$ disturbances according to Benjamin's (1963) classification, at the layer interfaces. One of Thorpe's hypotheses was that the instabilities will result in the production of a layer of mixed fluid. He made an attempt at exam-

\footnotetext{
*Received June 20, 2000. Accepted October 23, 2000.
}

ining the temporal variation of the layer thickness of this mixed portion but his results were inconclusive.

Later on Thorpe (1973), in a review of laboratory studies of mixing in stable stratified fluids, inspected available evidence on the evolution of the interface following the onset of the instabilities. He indicated that during the early stages of turbulence the interface grows rapidly and its structure is very variable, with very large density gradients and inversions. As time passes the interface thickness decreases and the density gradients and inversions become smaller. At the later stages the density gradient over the interface is almost constant but there are some micro-layers in the central region, better visible in a shadowgraph than on the density profile.

Turner (1973) examined Thorpe's experiments and, despite the above conflicting evidence, sug- 
gested that the interface should become well mixed after the growth of the instabilities. Turner warned about the possibility that the final density profile observed by Thorpe may be due either to the continuous acceleration of the flow, leading to the generation of new instabilities in the bounding interfaces, or to side effects, with turbulence generated at the solid boundaries continuously mixing both layers. Turner's hypothesis was supported by a number of atmospheric observations on the life cycle of $\mathrm{K}-\mathrm{H}$ billows and the way these billows cause the vertical temperature gradient to evolve (Scorer, 1969; Browning and Watkins, 1970).

Pelegrí and Sangrà (1998) have recently proposed a mechanism for the formation of mixed layers in stratified shear flow, which operates according to Turner's (1973) insight (see also Pelegrí and Csanady, 1994; Pelegrí et al., 1999; Rodríguez-Santana et al., 2001). Pelegrí and Sangrà assumed that mixing develops at the interface when the gradient Richardson number falls below a critical value. They showed that the statically very stable interface is a dynamically unstable region because of the strong velocity shear across it. A key point in their model is the incorporation of an equation that simulates the fact that turbulence, once generated, will last for some characteristic time even if the subcritical conditions disappear. Pelegrí and Sangrà (1998) were able to numerically simulate the conversion of an initial sharp interface into a mixed layer with adjacent sharp interfaces, and the progressive splitting of these interfaces leading to the formation of a staircase type structure.

In this work we will present the experimental setup and the results of several experiments that have been done to examine the evolution of the sheared interface. Our measurements essentially reproduce early efforts by Thorpe (1969, 1971, 1973) and other authors, illustrating the development and growth of K-H type instabilities. The experimental apparatus is considerably higher than the one used by Thorpe (1971), with the purpose of diminishing the side effects. We will pay particular attention to measuring the temporal evolution of the velocity in both layers, allowing us to determine the critical gradient Richardson number. Another contribution from this work is the assessment of the effective diffusivity prior to the development of the K-H type instabilities, an important value if we wish to make any comparison between our results and Pelegrí and Sangrà's (1998) numerical predictions.

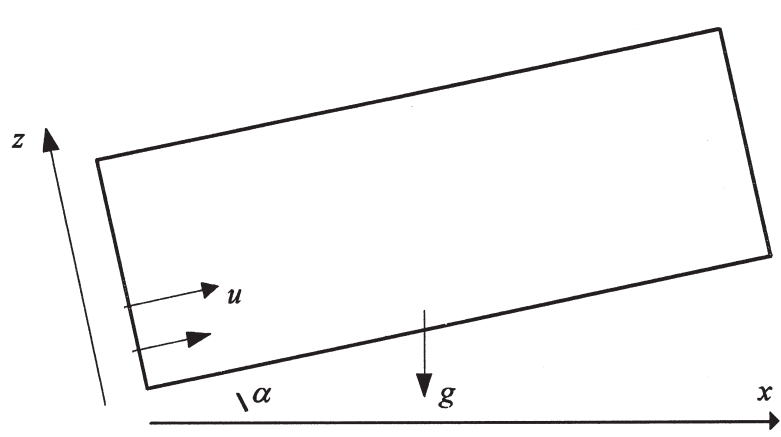

FIG. 1. - Notation for the theory of motion.

\section{THEORY OF MOTION}

Let us consider the motion of a stratified fluid from a state of rest between parallel planes, $z= \pm$ $H / 2$, inclined at an angle to the horizontal (Thorpe, 1971). The presence of boundaries is ignored and the $x$ and $y$ axes are taken parallel to the lateral walls (Fig. 1). The density of the fluid, $\rho$, is assumed to depend on the $z$ coordinate alone and, in accordance with observations, the velocity $(u, 0,0)$ is taken as parallel to the boundaries, a function of height $z$ and time $t$. For simplicity we shall suppose that the fluid is inviscid and neglect any dependence with the horizontal position. The equations of motion are:

$$
\rho \frac{\partial u}{\partial t}=\frac{\partial p}{\partial x}-g \rho \sin \alpha
$$

and

$$
0=\frac{\partial p}{\partial z}-g \rho \cos \alpha,
$$

where $p$ is pressure and $g$ is the gravity acceleration. The solution to these equations is

$$
u=-\frac{1}{\rho} \int_{0}^{t} \frac{\partial p}{\partial x} d t-g t \sin \alpha,
$$

after having used the condition $u(t=0)=0$.

Since the experimental tanks is closed there is no net flux across any constant $x$ plane so

$$
\int_{-H / 2}^{H / 2} u d z=0 .
$$

Combining equations (3) and (4) leads to

$$
\int_{0}^{t} \frac{\partial p}{\partial x} d t=\frac{-g H t \sin \alpha}{\int_{-H / 2}^{H / 2} \frac{1}{\rho} d z} .
$$

Substitution back into equation (3) gives: 


$$
u(z, t)=g t \sin \alpha\left[\left(\frac{H}{\rho} \int_{-H / 2}^{H / 2} \frac{1}{\rho} d z\right)-1\right] .
$$

If $\rho=\rho_{0}(1-\Delta f(z))$, where $\rho_{0}$ and $\Delta$ are constants, $\Delta<<1$, and $f(z)=-f(-z)$ is of order one, then (6) may be approximated by

$$
u=g \Delta f(z) t \sin \alpha .
$$

In our experiments the density profile is produced by allowing diffusion to occur for a time $\tau$ across an initially sharp interface that separates two homogeneous layers of brine and fresh water, with equal depths. The density distribution in the tank after a time $t$ is given by

$$
\rho=\rho_{0}\left[1-\Delta \operatorname{erf}\left(\frac{z}{2(K \tau)^{1 / 2}}\right)\right],
$$

provided that $H \gg 4(K \tau)^{1 / 2}$. The upper (fresh water) layer has uniform density $\rho_{0}(1-\Delta)$ and the lower (brine water) layer has a uniform density $\rho_{0}(1+\Delta)$, and $K$ is the molecular diffusivity of salt in water. With this density distribution and $\Delta<<1, t<\tau$, equation (7) may be approximated by

$$
u=g \Delta \operatorname{erf}\left(\frac{z}{2(K \tau)^{1 / 2}}\right) t \sin \alpha .
$$

\section{EXPERIMENTAL PROCEDURE}

\section{Experimental setup}

The apparatus is a closed rectangular tank with internal dimensions of $300 \mathrm{~cm}$ in length, $35 \mathrm{~cm}$ in height and $10 \mathrm{~cm}$ in width, and made of $0.8 \mathrm{~cm}$ thick glass (Fig. 2). The height of the apparatus is considerably greater than the one originally used by Thorpe (1971), which was of only $10 \mathrm{~cm}$ in height. One end is fitted with a tube that allows inlet and draining, and a plate that serves to arrest the inflow with the objective of reducing mixing while filling is done. The top has two other holes: one, located at the same end as the tube used to fill the tank, that allows air to leave, and another one located in the middle to allow conductivity-temperature (C-T) and velocity sensors to make profiles.

The tank is braced by a support system to prevent sagging and to allow pivoting around a horizontal axis located at a distance of $24 \mathrm{~cm}$ below its center. The ends of the tank are held with ridges, one of them of variable height to allow for changing the inclination angle.

The water is left to stand for some time, usually 24 hours, to remove air bubbles and to get a uniform temperature before using it to fill the tank. The tank is filled in a slightly inclined position, at about $1^{\circ}$

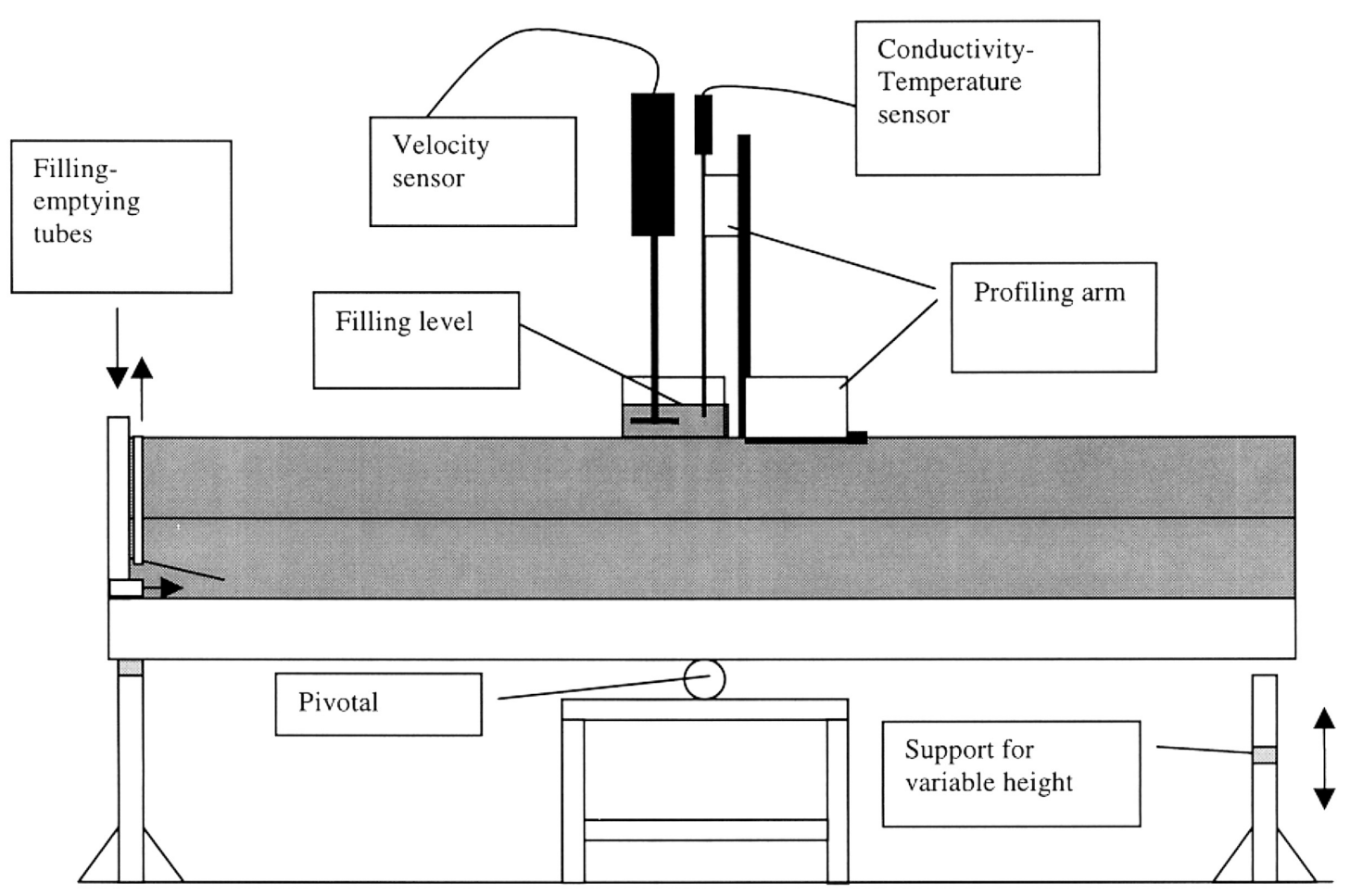

FIG. 2. - Schematics of the experimental apparatus. 
from the horizontal. The fresh water is first introduced at the lower end and the brine (and dyed) solution is next introduced, allowing it to slowly run under the fresh water. The slight inclination reduces mixing that otherwise would occur due to the downward slide of the brine solution. Filling is continued until the interface between the water and the brine reaches the middle of the rectangular tank, usually taking about 40-60 minutes. While filling is done the $1^{\circ}$ tank inclination is changed from one side to the other in order to help air leave.

After filling is finished the hole for releasing the air is closed and the tank recovers the horizontal position. At this point a video film of the experiment is started. The C-T sensor makes a first profile, very slowly such that the perturbation of the interface is minimized (about $16 \mathrm{~s}$ to profile a height of $30 \mathrm{~cm}$ ), and is left ready for a second profile. The tube is rapidly tilted a small angle, the layers accelerate in opposite directions, and the second C-T profile is rapidly done (less than $5 \mathrm{~s}$ for the whole profile). The C-T profile is done either before or after the development of the Kelvin-Helmholtz instabilities, depending on the objective of the experiment, but before the return wave (produced by reflection at the end of the tank) reaches the sampling position.

In most experiments the fractional density difference between the two fluids, $\Delta$, was chosen to be 0.085. In those experiments where $\Delta$ was much smaller the instability developed only when using large tilting angles, with the consequent difficulty of making the profiles and control the experiment. Even with this relatively large fractional density difference it is necessary to have a rather sharp interface to obtain a high velocity shear that produces a growing instability in a short period of time. The sharp interface was attained through the slow initial filling of the brine solution, using the technique described above, but reducing the total filling time such that diffusion is minimized.

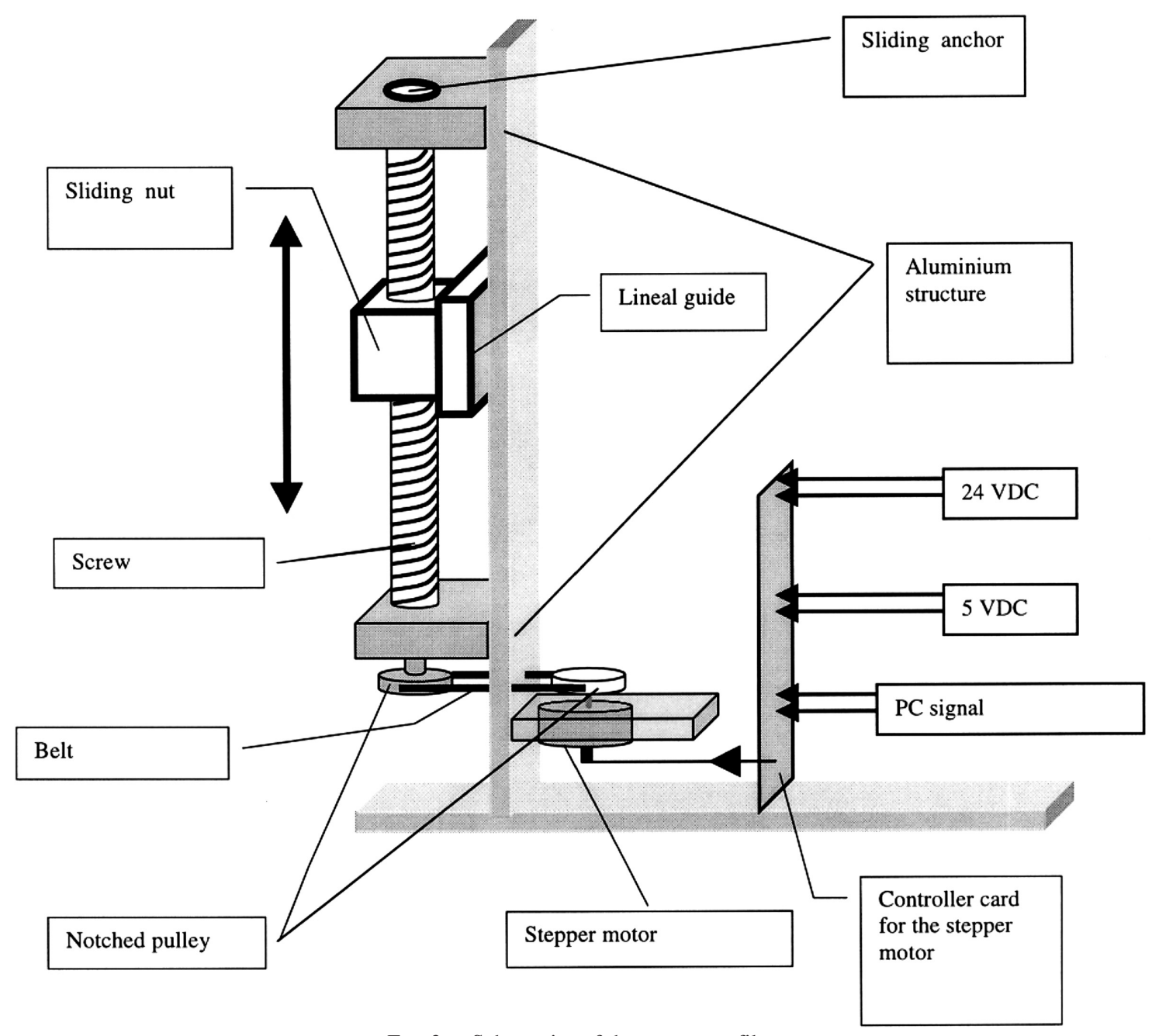

FIG. 3. - Schematics of the sensor profiler. 


\section{Profiler Description}

Two different instruments are used: a micro-scale C-T sensor (PME model $125 \mathrm{MSCTI}$ ) and a laboratory acoustic doppler velocity (Sontek model ADV). Two important aspects of these sensors are their small sampling size and rapid sampling rate. The head of the $\mathrm{C}$-T sensor samples over a distance less than 0.1 $\mathrm{mm}$, its sampling frequency is $800 \mathrm{~Hz}$ for conductivity and $143 \mathrm{~Hz}$ for temperature. The velocity sensor samples over a volume approximately $6 \mathrm{~mm}$ in diameter and its maximum sampling rate is $25 \mathrm{~Hz}$.
The profiler consists of a mechanical gear used to introduce the C-T and velocity sensors into the tank. The mechanical part consists in a stepper motor connected to a vertical arm where the sensors are attached (Fig. 3). The stepper motor is controlled through a personal computer, and the data measured with the sensors is also transferred to the computer (Fig. 4).

The stepper motor turns step by step, as the motor driver receives a square wave signal input. This means that the number of rotations, as well as the velocity of the motor, is perfectly controlled

HARDWARE SCHEME

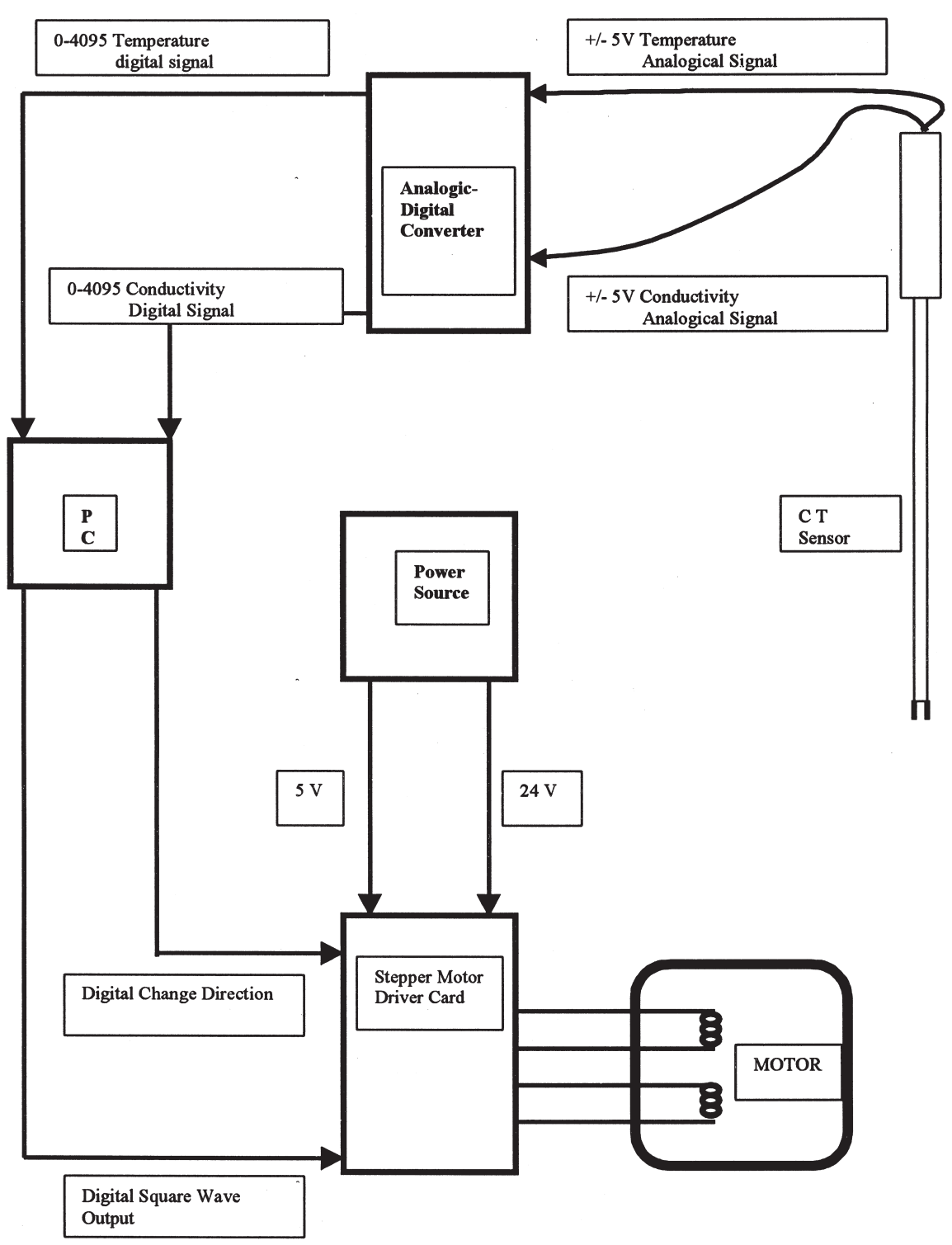

FIG. 4. - Schematics of the data acquisition system. 
through the frequency of the input signal. The stepper motor turns a screw connected to a lineal guide through a nut designed to minimize friction and horizontal movements. In this manner the sensors, attached to the guide, have a very good vertical resolution. This resolution was determined, through a catetometer, to be approximately $0.1 \mathrm{~mm}$.

The software is designed to take temperature and conductivity data as the motor driver receives the square wave signal or, equivalently, as the motor turns each step. Since each step means vertical distance (approximately $0.104 \mathrm{~mm}$ ) this provides a profile of these variables against depth.

\section{EXPERIMENTAL RESULTS}

Figure 5 illustrates the four principal steps in any experiment as seen with pictures taken with the video camera: an initial density profile, tilting of the tank and flow acceleration, onset and growth of the K-H instabilities, and a final density profile. Several specific measurements done during the experiments are discussed next.

\section{Effective diffusivity}

As the experiment is set up the interface between the two fluid layers will become diffused. If the tank is filled very carefully, such that no turbulence is induced, the diffusion is a molecular process and the only important parameter will be the time required to fill the tank with the brine solution (some 20-30 $\min$ ), what we could call the diffusive time $\tau$. In this case the initial size of the interface would be of order $(K \tau)^{1 / 2}$, where $K=1.4 \times 10^{-9} \mathrm{~m}^{2} \mathrm{~s}^{-1}$ is the molecular diffusivity of salt (Batchelor, 1967).

In practice, however, the salt diffusivity during the experimental setup will be larger than the molecular value because of the generation of a small amount of turbulence when introducing the fluids. To estimate this laboratory molecular diffusivity we have made several measurements of the density profile after filling the tanks, as well as additional measurements on the evolution of the density profile in cylindrical containers initially filled with a twolayer solution. We have found that the best adjustment to the observations, using a density distribution given by equation (8), corresponds to a laboratory diffusivity of $2.2 \times 10^{-8} \mathrm{~m}^{2} \mathrm{~s}^{-1}$, or equivalently, to the following approximate equation for the interface thickness:

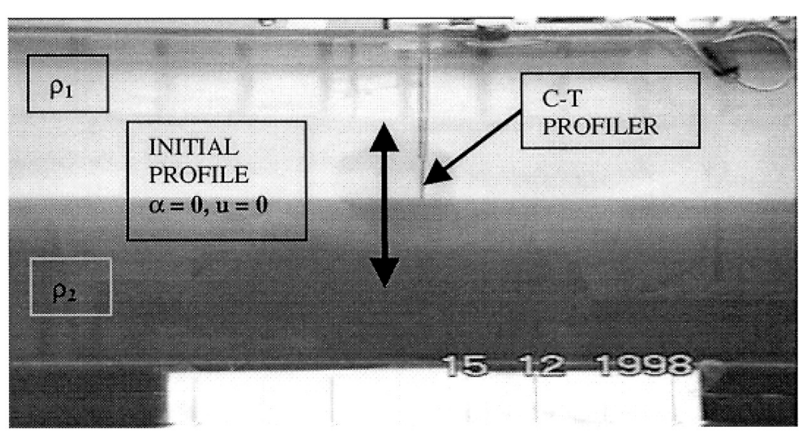

(a) Initial profile.

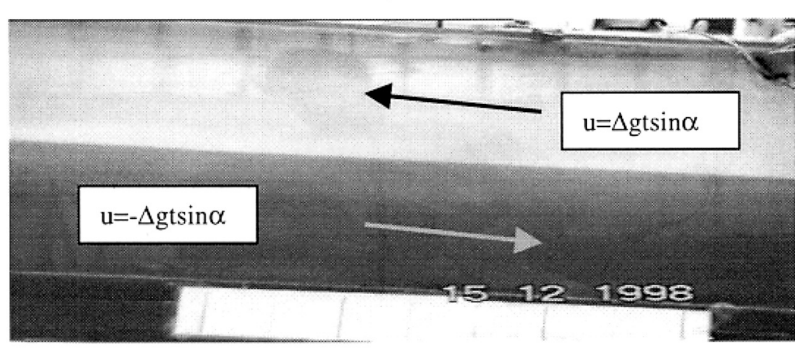

(b) Starting the experiment.

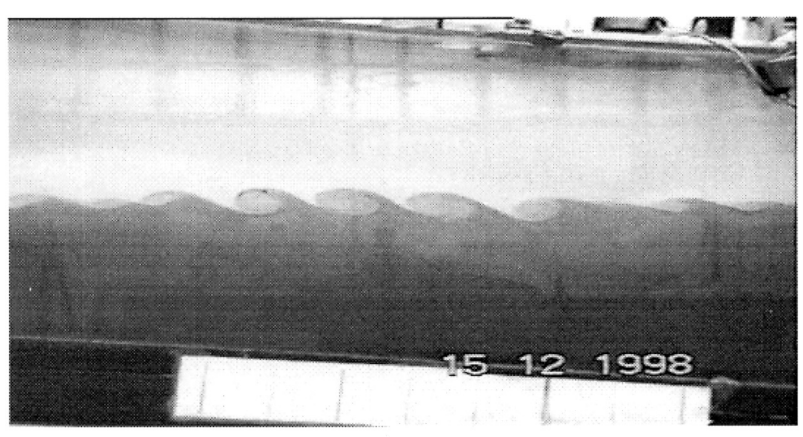

(c) Kelvin-Helmholtz instability.

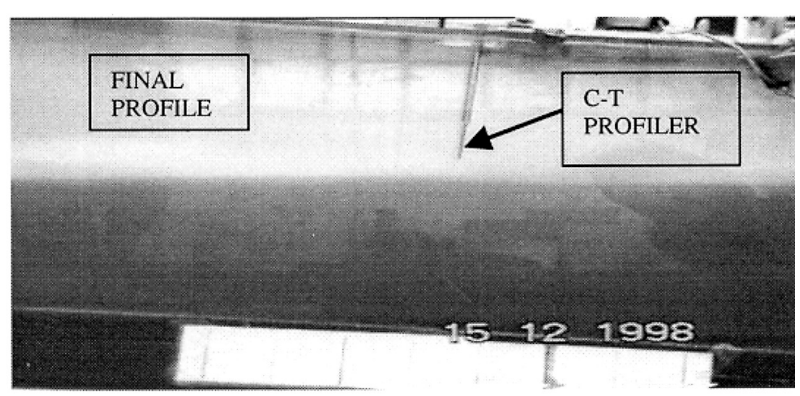

(d) Final profile.

FIG. 5. - Pictures of principal steps in an experiment as obtained with the video camera.

$$
\delta z=4(K \tau)^{1 / 2}
$$

This equation shows that, after filling the tank, a reasonable estimate of the interface thickness is 5 $\mathrm{mm}$. Figure 6 compares one actual density profile prior to tilting the tank, as determined from the con- 


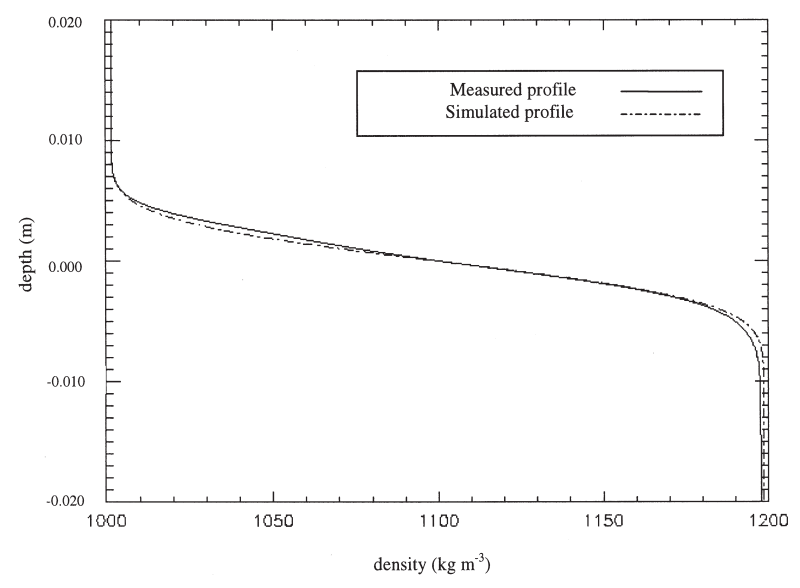

FIG. 6. - Comparison of measured and simulated profiles after filling the tank.

ductivity and temperature measurements, with the density profile obtained from equation (8) and using a laboratory diffusivity of $2.2 \times 10^{-8} \mathrm{~m}^{2} \mathrm{~s}^{-1}$.

An important aspect to take into account is that the actual size of the interface, after the flow has accelerated and just before the K-H instability develops, is even larger, typically by a factor of four. The reason for this is that from the time when the tank is tilted to immediately before the onset of the $\mathrm{K}-\mathrm{H}$ instabilities the flow accelerates and the actual diffusivity is much larger than the molecular diffusivity. Using measurements of the conductivity profile just before the onset of the K-H instabilities, with $\tau$ now being the time elapsed since the tank was tilted plus half the time that it takes the tank to tilt, equation (10) gives an effective diffusivity of $4 \times 10^{-7} \mathrm{~m}^{2} \mathrm{~s}^{-1}$.

\section{Velocity evolution}

The non-intrusive acoustic velocity sensor provides a very useful way to check Thorpe's (1971) theory of motion, which was not available at his time. Thorpe (1971) predicted that the velocity distribution at a time $t$ after the tube has been tilted is quite accurately given by equation (7). In all our experiments $\Delta<0.1$ and $t / \tau<0.01$ so expression (9) should also provide a good approximation to the temporal evolution of the velocity field. At distances relatively away from the initial position of the interface $(z=0)$ equation (9) gives

$$
u=g t \Delta \operatorname{sen} \alpha .
$$

Thorpe's (1971) theory of motion assumes that at the initial time, $t=0$, the flow starts accelerating

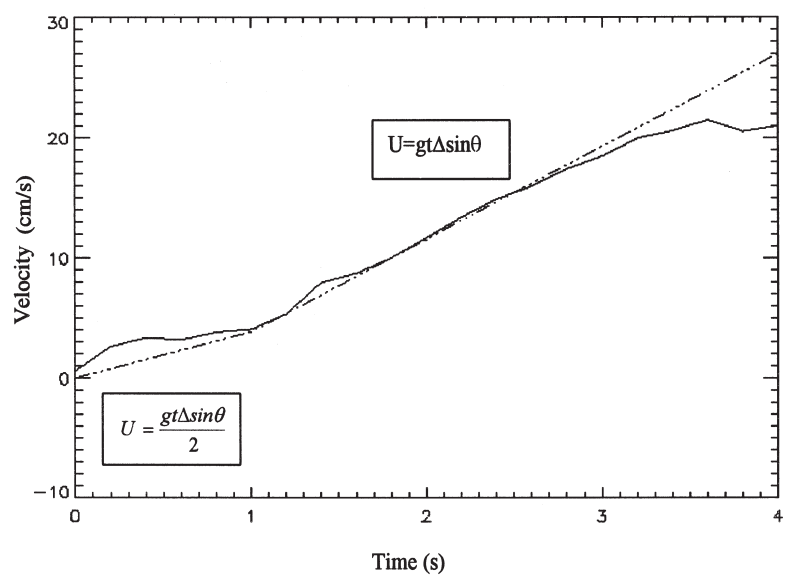

FIG. 7. - Comparison of measured and simulated velocities in one layer, relatively away from the interface.

according to equation (9). In practice, however, the rectangular tank takes some time to reach its maximum inclination so a time offset, which depends on how fast the rectangular tank is tilted, is introduced. Since the tank is usually tilted in less than half a second the offset in the time origin will be of order 0.1 s. Another possibility is to use a modified equation (11) while tilting the tank, as discussed next.

Figure 7 compares the velocity in the upper layer (measured at $10 \mathrm{~cm}$ from the interface) and the velocity given by expression (11), for the case with $\alpha=5^{\circ}$ and $\Delta=0.091$. In this experiment the time required to tilt the tank was $1 \mathrm{~s}$. During the first second (approximately what takes the tank to be tilted) we may appreciate that the slope of the measured velocity increases more slowly. This was reasonably well simulated by simply taking the velocity as given by half the value of equation (11).

The agreement between the measured and expected velocities is reasonably good during the first three seconds, after which the measured velocity increases more slowly. At this time mixing has greatly increased due to the developing instabilities and Thorpe's (1971) theory of motion will not be valid anymore. Diapycnal mixing clearly redistributes not only mass but also momentum, in this case bringing lower layer momentum (with opposite sign) to the upper layer.

\section{Instability development}

The main parameter that controls the onset of K$\mathrm{H}$ instabilities is the gradient Richardson number, directly proportional to the vertical density gradient $\partial \rho / \partial z$ and inversely proportional to the square of the vertical velocity gradient $\partial u / \partial z$ : 


$$
R i=\frac{g\left(\frac{\partial \rho}{\partial z}\right)}{\rho\left(\frac{\partial u}{\partial z}\right)^{2}}
$$

In laboratory experiments, however, it is usual to use instead the initial Richardson number, defined in terms of the thickness of the initial interface $\delta z$, the density across the interface $\Delta \rho$, and the velocity difference across the interface $U$, as follows:

$$
R i=\frac{g \Delta \rho \delta z}{\rho U^{2}}
$$

A detailed description of the generation of shear instability and its dependence on the initial Richardson number is available in a number of review papers, such as Thorpe (1987), Gregg (1987) and Fernando (1991).

The instability at the interface develops at about the same time all along the central portion of the tube. As indicated by Thorpe $(1971,1973)$ the K-H type instabilities grow very rapidly in time until they break. Both the time required for the instability to break and the size of the instability are inversely proportional to the density gradient at the interface, i.e. by increasing the thickness of the initial interface the K-H instabilities become larger and take longer to break.

Figure $8 \mathrm{a}$ illustrates the lineal relation present between the thickness of the initial interface and the time at which the K-H instability occurs. With the help of velocity measurements (Fig. 7) we may draw a relationship between the thickness of the initial interface and the Richardson number at the onset of the instability. The Richardson number is calculated following equation (13), with the initial density difference, the size of the interface prior to the instability as given by equation (10), and the velocity difference according to equation (11). Figure $8 \mathrm{~b}$ shows that the relationship between the thickness of the initial interface and the Richardson number gives a critical value close to 0.25 , although this value appears to be inversely related to the size of the initial interface.

\section{Layer structure}

The instability produces a very rapid movement of the fluids near the interface, bringing the heavier fluid up and vice versa, causing the appearance of some regions of near-homogeneous density bounded by inversions (Fig. 9a). This movement, however, appears to not produce a definitive mixture of all fluids involved, and as time passes the fluids are sorted out by their own density. It is important to note that sampling is limited by the time that it takes for a wave reflected at the end of the tank to reach its central portion, typically of the order of $10 \mathrm{~s}$.

One striking aspect is that the size of the interface, defined as the region where density has suffered a significant modification, is much larger immediately after the generation of the K-H insta-
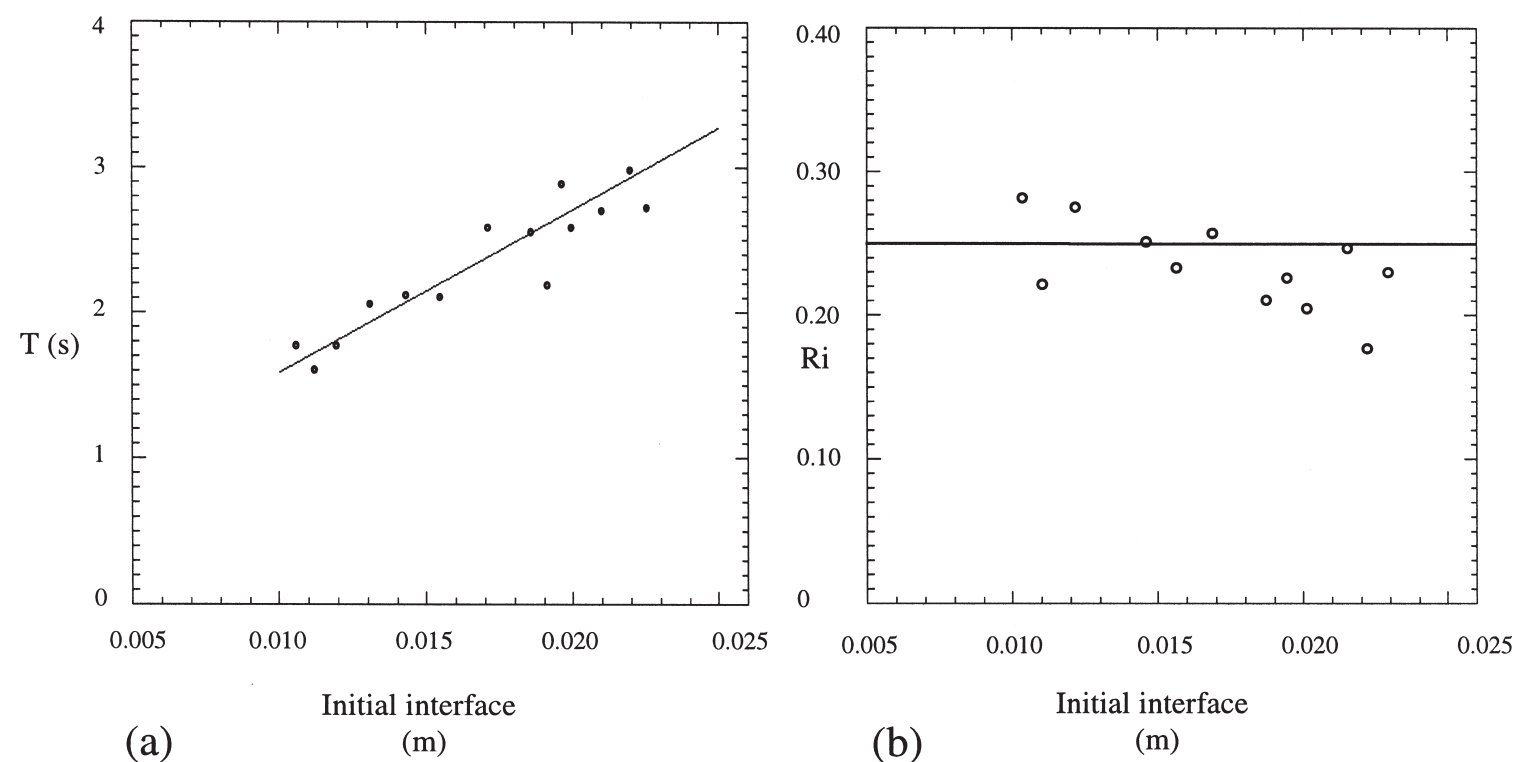

FIG. 8. - (a) Time for the onset of the K-H instability as a function of the initial interface thickness. (b) Richardson number when the K-H instability develops as a function of the initial interface thickness. 

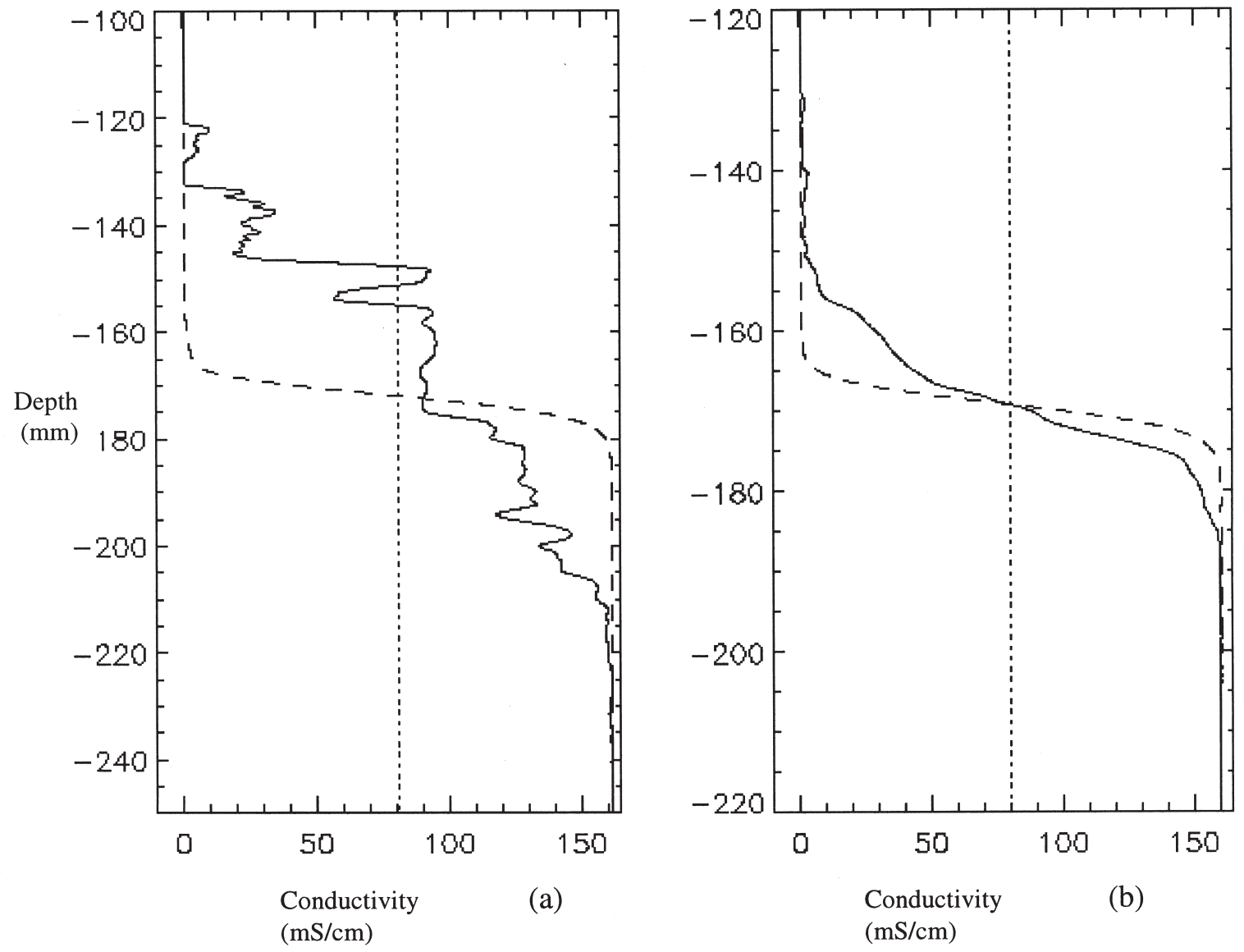

FIG. 9. - Conductivity profiles (a) half a second after the onset of the K-H instability and (b) seven seconds after the onset of the K-H instability.

bilities than a few seconds later. After a few seconds the interface thickness has reduced and any layered structure is of much smaller size than the original near-homogeneous regions (Fig. 9b). This feature was already noticed by Thorpe (1973) and was ascribed to possible lateral side effects. In our experiments the vertical size of the tank is much larger and the results are strikingly similar, both in terms of the temporal and vertical evolution of the interface. This confirms that the upper and lower walls do not control the redistribution of the density layers.

We hypothesize that the final density structure depends on the existence of other mechanisms capable of producing additional mixing while the inversions are present, before the water layers have time to sort out by density. In the type of experiments here presented the $\mathrm{K}-\mathrm{H}$ instabilities are very small and fast, and it is difficult to think of other mechanisms that can induce sufficient collateral mixing. In geophysical flows, however, the $\mathrm{K}-\mathrm{H}$ instabilities may be sufficiently large in size and time that other mixing processes become important.

\section{CONCLUSIONS}

We have presented an experimental methodology and apparatus to examine the onset and growth of $\mathrm{K}$ $\mathrm{H}$ instability in stratified shear flow. Our results resemble previous experimental efforts by Thorpe $(1969,1971,1973)$ but include the assessment of the effective diffusivity prior to the development of the $\mathrm{K}-\mathrm{H}$ instability and the measurement of the velocity field. From these results we can better quantify the conditions for the onset of the K-H instability. This turns out to take place when the Richardson number is close to 0.25 , although this value appears to slightly decrease with an increasing thickness of the initial interface.

Our results do not reproduce the large layer structure proposed by Turner (1973) or, more recently, by Pelegrí and Sangrà (1998). We hypothesize that the final structure must depend on the time available for other mixing processes to take place, while $\mathrm{K}-\mathrm{H}$ billows are active and before the water layers are sorted out by their own density. 


\section{ACKNOWLEDGEMENTS}

This work has been supported by the Spanish government through CICYT's grant number MAR96-1893.

\section{REFERENCES}

Batchelor, G.K. - 1967. An introduction to Fluid Dynamics. Cambridge University Press, Cambridge, $615 \mathrm{pp}$.

Benjamin, T.B. - 1963. The threefold classification of unstable disturbances in flexible surfaces bounding inviscid flows. J. Fluid Mech., 16: 436-450.

Browning, K.A. and C.D. Watkins. - 1970. Observations of clear air turbulence by high power radar. Nature, 227: 260-263.

Fernando, H.J.S. - 1991. Turbulent mixing in stratified fluids. Ann. Rev. Fluid Mech., 23: 455-493.

Gregg, M.C. - 1987. Diapycnal mixing in the thermocline: A review. J. Geophys. Res., 92: 5249-5286.

Pelegrí , J.L. and G.T. Csanady. - 1994. Diapycnal mixing in western boundary currents. J. Geophys. Res., 99: 18275-18304.
Pelegrí, J.L. and P. Sangrà. - 1998. A mechanism for layer formation in stratified geophysical flows. J. Geophys. Res., 103: 30679-30693

Pelegrí, J.L., A. Rodríguez-Santana, P. Sangrà and A. MarreroDíaz. - 1998. Modeling of shear-induced diapycnal mixing in frontal systems. App. Sci. Res., 59: 159-175.

Rodríguez-Santana, A., J.L. Pelegrí, P. Sangrà and A. MarreroDíaz. - 1999. Diapycnal mixing in Gulf Stream meanders. $J$. Geophys. Res., 104: 25891-25912.

Rodriguez-Santana, A., J.L. Pelegrí, P. Sangrà and A. MarreroDíaz. - 2001. On the relevance of diapycnal mixing for the stability of frontal meanders. Sci. Mar., 65(Suppl. 1): 259-267.

Scorer, R.S. - 1969. Billow mechanics. Radio Sci., 4: 1299-1308.

Thorpe, S.A. - 1969. Experiments on the instability of stratified shear flows: inmiscible fluids. J. Fluid Mech., 39: 25-48.

Thorpe, S.A. - 1971. Experiments on the instability of stratified shear flows: miscible fluids. J. Fluid Mech., 46: 299-319.

Thorpe, S.A. - 1973. Turbulence in stably stratified fluids: A review of Laboratory experiments. Boundary Layer Meteor., 5: 95-119.

Thorpe, S.A. - 1987. Transitional phenomena and the development of turbulence in stratified fluids: A review. J. Geophys. Res., 92: 5231-5248.

Turner, J.S. - 1973. Buoyancy effects in fluids. Cambridge University Press, Cambridge, 367 pp. 